\title{
Acquired fear, brightness preference, and one-way shuttlebox performance'
}

\section{JAMES ALLISON, DANIEL LARSON, AND DONALD D. JENSEN}

INDIANA UNIVERSITY

Rats choosing between black and white showed an unlearned preference for black which was strengthened by previous shock in white, and weakened but not reversed by equivalent shock in black. Congruous findings were obtained with a one-way shuttlebox: escape from white to black occurred more readily than escape from black to white, whether $S$ had or had not been shocked in the start compartment. Incongruously, fear conditioning did not facilitate escape performance, but rather impaired it early in training.

In the classic demonstration of an acquired fear drive, rats shocked in the white side of a shuttlebox later learned to escape from the white to the black side, although both sides were then free of shock (Miller, 1948). Those data suggest that rats previously shocked in white would prefer black to white, given a choice between the two-as do rats with no history of shock (Douglas, 1966; Freedman, 1965). Further, it seems likely that the unlearned preference for black would be strengthened by shock in white, and weakened or even reversed by shock in black, but Miller's data provide no basis for these predictions. There was no nonshocked control group, and no group run from black to white. Later work with the Miller shuttlebox does not seem to include any factorial experiment using the four relevant conditions: escape from white to black or from black to white after preliminary experience in the start compartment with or without shock. The two experiments reported here were done (a) to confirm the preference for black over white among rats with no history of shock, (b) to study the extent to which the preference could be increased by previous shock in white, and decreased by previous shock in black, and (c) to examine the relationship between brightness preference and instrumental performance in the Miller shuttlebox.

The preference tester was a wooden enclosure 14 in. high, with a $25 \times 25$ in. grid floor and a frosted Plexiglas ceiling. Partition walls divided it into a start compartment, $12 \times 25$ in., and two alternative goal compartments, $12 \times 12$ in. each. Start and goal compartments were separated by a guillotine door, $9 \times 14$ in. An open passageway between the two goal compartments allowed $S$ to move back and forth between them after the initial choice had been made. Wall and floor colors were manipulated by means of interchangeable wall inserts and floor pans painted black or white, and interchangeable guillotine doors were painted appropriately. $S$ chose between a white and a black goal compartment after preliminary experience in the start compartment, white or black, with or without shock.
A total of 40 male Long-Evans rats, 90-150 days old, were assigned randomly to four groups of 10: WNS (white start compartment, no shock), WS (white start compartment, shock), BNS (black start compartment, no shock), or BS (black start compartment, shock). On Day $1, \mathrm{~S}$ either was or was not shocked while confined for $7 \mathrm{~min}$ in the start compartment with the guillotine door closed. The shock treatment consisted of 24 1-sec, 1-mA scrambled shocks at one every $15 \mathrm{sec}$, beginning with the second minute of confinement. Shock invariably elicited vocalization and startle. $S$ was then removed to a holding cage for $10 \mathrm{~min}$, and returned to the home cage. On Day 2, 24 h later, $\mathrm{S}$ got five choice trials with an intertrial interval of $4 \mathrm{~min}$. Half of each group had the black goal compartment on the right, half on the left. $E$ began each trial by placing $S$ in the start compartment through a doorway centered laterally in its long wall. E raised the guillotine door after a 15-sec delay, closed it when $S$ entered either goal compartment, and removed $S$ to the holding cage 100 sec later. On each trial, E recorded initial choice and time spent in each goal compartment.

As Table 1 shows, nonshocked Ss chose the black goal compartment more often than the white (twotailed, $p=.04$ by the binomial test). The preference for black over white was increased by previous shock in white, and decreased but not reversed by previous shock in black, producing a significant interaction ( $F=$ 5.64, $\mathrm{df}=1 / 36, \mathrm{p}<.025$ ). Time spent data agreed closely with initial choice data. Nonshocked Ss spent a greater proportion of time in the black than the white goal compartment (two-tailed, $p=.02$ ). The preference for black over white was increased by shock in white, and decreased by shock in black $(F=7.96, \mathrm{df}=1 / 36, \mathrm{p}<.01$ ). There were no significant effects involving the trials variable.

Four corresponding groups were trained in a shuttlebox to escape from white to black or from black to

Table 1

Initial choice of black rather than white, and time spent in black rather than white.

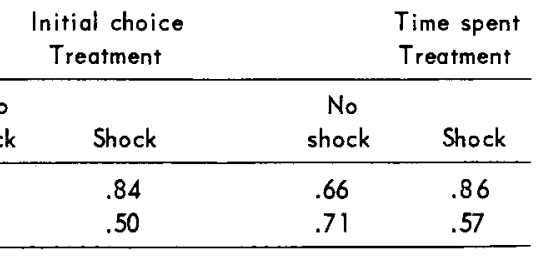

The tabular entries are mean proportions based on all five trials. 


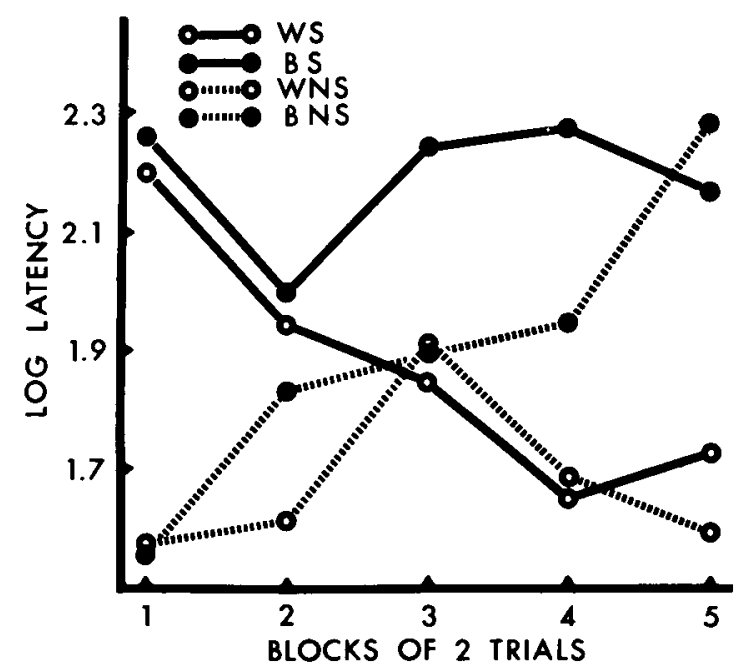

Fig. 1. Mean log latency (sec) in blocks of two trials.

white after preliminary experience in the start compartment with or without shock. Since rats with no history of shock preferred black to white, we expected Group WNS to perform better than Group BNS. Since previous shock in white increased the preference for black over white, and equivalent shock in black decreased it by a comparable amount, Group WS was expected to perform better than Group BS. Similarly, Group WS was expected to perform better than WNS, and BS better than BNS.

The Ss were male Long-Evans rats, 90-150 days old, assigned randomly to four groups of nine. The shuttlebox was patterned closely after Miller's, except that ours had a grid floor on each side, with a black or white floor pan. On Day $1, \mathrm{~S}$ was confined in the black or white side for $7 \mathrm{~min}$, with or without $241-\mathrm{sec}, 1-\mathrm{mA}$ scrambled shocks. On Day 2, 24 h later, $\mathrm{S}$ was trained to escape from the appropriate start to the appropriate goal compartment by touching the paddlewheel just above the guillotine door, with either forepaw. When the wheel was touched the door was opened, and $\mathrm{S}$ spent $30 \mathrm{sec}$ in the goal compartment before being removed to the holding cage. Each $S$ got 10 trials, with an intertrial interval of $2 \mathrm{~min}$.

Two procedural differences between our experiment and Miller's should be pointed out. First, Miller's Ss received both escapable shock and inescapable shock during the fear conditioning phase. We did not use escapable shock because we were unable to adapt an escape procedure to our nonshocked controls without introducing other contaminating differences between their treatment and the shock treatment. Second, Miller discarded several Ss for failing to respond within a $100-$ sec cutoff early in escape training. We discarded none.
As expected, Group WNS performed better than Group BNS (see Fig. 1). Group WNS showed no consistent trend as training progressed $(F=1.16, d f=4 / 64, p>.25)$, while BNS responded progressively slower across trial blocks ( $F=3.90, \mathrm{df}=4 / 64, \mathrm{p}<.01)$. As expected, WSperformed better than BS. Group WS responded progressively faster as training progressed ( $F=3.77, d f=4 / 64, p<$ .01 ), confirming Miller's findings, while BS showed no consistent trend ( $F=1.26$, df $=4 / 64, p>.25$ ).

According to acquired drive theory, and according to our preference data, WS should have performed better than WNS, and BS better than BNS. These predictions could not be evaluated unambiguously. At the outset of escape training, WS performed worse than WNS $(t=2.13, d f=16$, two-tailed, $p<.05)$, and BS worse than BNS ( $t=3.55, \mathrm{df}=16$, two-tailed, $p<.01)$. At the end of training WS did not differ from WNS, nor BS from BNS. Thus, the fear conditioning procedure may simply have produced a temporary depression of performance among WS and BS, and no effect upon the reinforcing properties of escape from white to black or from black to white, respectively.

We conducted a separate analysis after excluding five Ss that would have been discarded had we adopted Miller's cutoff criterion (Miller, 1948, pp. 92-93). The analysis required no change in the conclusions presented above.

Most textbooks attribute Miller's results-the progressive improvement in escape performance-to acquired reinforcing properties of escape from white to black. This work suggests that such an interpretation is misleading and oversimplified in two respects. First, it seems clear that fear conditioning depresses initial performance in the shuttlebox. Consequently, it is uncertain how much of the subsequent improvement in escape performance can be attributed to reinforcement. Second, it seems clear that the fear conditioning procedure operates upon an unlearned preference for black over white. Consequently, if Miller's rats were reinforced by escape from white to black, its reinforcing properties were probably not entirely acquired.

\section{References}

Douglas, R. J. Cues for spontaneous alternation. J. comp. phy siol. Psychol, 1966, 62, 17 1-183.

Freedman. P. E. Habituation of alternation behavior. J. exp. Psychol., 1965, 69, 613-617.

Miller, N. E. Studies of fear as an acquirable drive: I. Fear as motivation and fear-reduction as reinforcement in the learning of new responses. J. exp. Psychol., 1948, 38, 89-101.

\section{Note}

1. This work was supported in part by Grant MH 11470 from the National Institute of Mental Health, United States Public Health Service, to J. A. The participation of D. L. was supported by NSF Undergraduate Research Participation Program Grant GY 878. We thank William C. Leonard for his assistance. 